\title{
Calcineurin suppresses AMPK-dependent cytoprotective autophagy in cardiomyocytes under oxidative stress
}

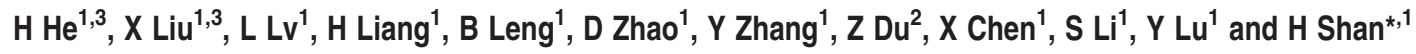

Calcineurin signalling plays a critical role in the pathogenesis of many cardiovascular diseases. Calcineurin has been proven to affect a series of signalling pathways and to exert a proapoptotic effect in cardiomyocytes. However, whether it is able to regulate autophagy remains largely unknown. Here, we report that prolonged oxidative stress-induced activation of calcineurin contributes to the attenuation of adaptive AMP-activated protein kinase (AMPK) signalling and inhibits autophagy in cardiomyocytes. Primary cardiomyocytes exhibited rapid formation of autophagosomes, microtubule-associated protein 1 light chain 3 (LC3) expression and phosphorylation of AMPK in response to hydrogen peroxide $\left(\mathrm{H}_{2} \mathrm{O}_{2}\right)$ treatment. However, prolonged $(12 \mathrm{~h}) \mathrm{H}_{2} \mathrm{O}_{2}$ treatment attenuated these effects and was accompanied by a significant increase in calcineurin activity and apoptosis. Inhibition of calcineurin by FK506 restored AMPK function and LC3 expression, and decreased the extent of apoptosis caused by prolonged oxidative stress. In contrast, overexpression of the constitutively active form of calcineurin markedly attenuated the increase in LC3 induced by short-term $(3 \mathrm{~h}) \mathrm{H}_{2} \mathrm{O}_{2}$ treatment and sensitised cells to apoptosis. In addition, FK506 failed to induce autophagy and alleviate apoptosis in cardiomyocytes expressing a kinase-dead K45R AMPK mutant. Furthermore, inhibition of autophagy by 3-methylanine (3-MA) or by knockdown of the essential autophagy-related gene ATG7 abrogated the protective effect of FK506. These findings suggest a novel role of calcineurin in suppressing adaptive autophagy during oxidative stress by downregulating the AMPK signalling pathway. The results also provide insight into how altered calcineurin and autophagic signalling is integrated to control cell survival during oxidative stress and may guide strategies to prevent cardiac oxidative damage.

Cell Death and Disease (2014) 5, e997; doi:10.1038/cddis.2013.533; published online 16 January 2014

Subject Category: Experimental Medicine

Oxidative stress is mainly caused by a disturbance to the basal cellular redox state and has been implicated in various human diseases, ${ }^{1,2}$ especially heart pathophysiological processes. It results in damage to subcellular organelles and nucleic acids and causes protein aggregation as well as alternations to a sequence of signalling cascades that affect cell apoptosis, ageing and autophagy.,4 Studying the mechanisms underlying the interaction of these signals in the context of cardiomyocyte oxidative stress would enable us to gain a deeper understanding of how to prevent oxidative damage during cardiovascular complications such as ischaemia-reperfusion injury.

Cardiomyocytes are highly metabolic and nonreplicative cells for which maintenance of homeostasis largely depends upon intracellular renewal. Autophagy refers to an evolutionarily conserved and programmed self-degrading process during which damaged organelles and aggregated proteins are enveloped by double membrane vesicles and sequestered for lysosome degradation. ${ }^{5,6}$ Recent studies have highlighted the importance of autophagy in both physiological and pathological states. It is widely accepted that a physiological level of autophagy is crucial for maintenance of cellular homeostasis, whereas cells that undergo excessive autophagy exhibit autophagic cell death. Despite reports that autophagy can execute cell death in various scenarios, recent accumulating evidence has demonstrated that autophagy is more of a survival-prone response than an inducer of cell death. ${ }^{6,7}$ Autophagy enables cells to recycle aggregated proteins and damaged organelles into raw materials that is essential for living cells to recover from

\footnotetext{
${ }^{1}$ Department of Pharmacology (State-Province Key Laboratories of Biomedicine- Pharmaceutics of China, Key Laboratory of Cardiovascular Research, Ministry of Education), Harbin Medical University, Harbin, Heilongjiang, People's Republic of China and ${ }^{2}$ Institute of Clinical Pharmacy, The Second Affiliated Hospital, Harbin Medical University, Harbin, Heilongjiang 150081, People's Republic of China

${ }^{*}$ Corresponding author: H Shan, Department of Pharmacology, Harbin Medical University, No. 157, Baojian Road, Harbin, Heilongjiang 150081, People's Republic of China. Tel: + 86451 86671354; Fax: + 86451 86671354; E-mail: shanhongli72@aliyun.com

${ }^{3}$ These authors contributed equally to this work.

Keywords: oxidative stress; cardiomyocyte; autophagy; calcineurin; AMPK

Abbreviations: AMPK, AMP-activated protein kinase; LC3, microtubule-associated protein 1 light chain 3; CnA, calcineurin subunit A; ATG, autophagy-related proteins; mTOR, mammalian target of rapamycin; Akt, protein kinase B; p70S6K, p70 ribosomal protein S6 kinase; 4E-BP1, elongation factor 4E binding protein 1; FOXO, Forkhead box; TAK1, TGF- $\beta$-activated kinase 1; LKB1, liver kinase B1; CaMKK $\beta$, calcium/calmodulin-dependent protein kinase kinase $\beta$; GFP, green fluorescent protein; mRFP, monomeric red fluorescent protein; DN, dominant negative; ROS, reactive oxygen species; PBS, phosphate-buffered saline; DMSO, dimethyl sulphoxide; 3-MA, 3-methyladenine; FBS, fetal bovine serum; TUNEL, terminal deoxynucleotidyl transferase dUTP nick-end labelling; DAPI, 4',6-diamidino-2phenylindole; BrdU, 5-Bromo-2-deoxyUridine; siRNA, small interfering RNA

Received 26.8.13; revised 24.11.13; accepted 02.12.13; Edited by RA Knight
} 
stresses such as energy depletion and oxidative and endoplasmic reticulum stress (ER stress)..$^{8-11}$ Some diseases, including diabetes, cancer and neurodegenerative diseases, are closely related to malfunctions in autophagy. ${ }^{6,12,13}$ Moreover, genetically depleting autophagy disrupts cellular functions in vivo and in vitro. ${ }^{14-16}$ These reports suggest that autophagy may be a versatile and essential survival mechanism against stressors in mammalian cells.

The nutrient sensor mTOR (mammalian target of rapamycin) is likely the core regulator of autophagy. It suppresses autophagy through inactivation of ATG1 and ATG13 that are indispensable autophagy-related proteins (ATGs) for autophagosome biogenesis. ${ }^{17,18}$ In parallel, the energy sensor AMPK (5' adenosine monophosphate-activated protein kinase), which is abundant in cardiomyocytes, can activate autophagy through direct activation of ULK1 or by inhibiting mTORC1 in mammalian cells. ${ }^{19,20}$

AMPK is a kinase composed of the catalytic subunit $\alpha$ and the regulatory subunits $\beta$ and $\gamma$. AMPK becomes activated when phosphorylation occurs at threonine-172 of the catalytic subunit. $^{21}$ It modulates cell functions by regulating cell pathophysiological processes such as apoptosis, lipid metabolism, ageing and autophagy under stress. ${ }^{20,22}$ Despite proof that AMPK protects cardiomyocytes from oxidative stress-induced injury, ${ }^{23-25}$ little is known about the role of AMPK pathway-mediated autophagy in cardiomyocytes under oxidative stress. In addition, given the various altered signalling cascades during oxidative stress, it is tempting to assume that some signals might regulate autophagy by interacting with the AMPK pathway.
Calcineurin, also known as calcium/calmodulin-dependent protein phosphatase 2B (PP2B), reportedly mediates multiple signalling cascades, including apoptosis, by dephosphorylating several substrates in cardiovascular diseases. ${ }^{26-28}$ It is still a matter of debate whether calcineurin is protective or detrimental to the heart. In addition, it is unknown whether calcineurin participates in regulating autophagy of cardiomyocytes during oxidative stress. The pharmacological inhibitor of calcineurin, cyclosporine A, has been reported to enhance AMPK phosphorylation in the rat hippocampus, ${ }^{29}$ indicating the potential role of calcineurin in autophagy regulation. Therefore, we explored whether calcineurin is capable of regulating autophagy and the underlying molecular mechanism. Our present study reveals a novel role of calcineurin as an autophagy inhibitor by repression of the AMPK/mTOR signalling pathway. Moreover, we demonstrate that AMPK-dependent autophagy is cytoprotective in the context of oxidative stress.

\section{Results}

Oxidative stress induces apoptosis and autophagy and increases calcineurin activity in cardiomyocytes. In this study, we first confirmed the presence of oxidative damage in primary cardiomyocytes. We assessed cell viability at various time points using MTT assay. As shown in Figure 1a, there was a time-dependent decrease in cell viability to $40 \%$ compared with the control group at $12 \mathrm{~h}$ after exposure to hydrogen peroxide $\left(\mathrm{H}_{2} \mathrm{O}_{2}\right)$. The reduction in cell viability was significantly reversed by pan-caspase inhibitor zVAD-fmk at later time points ( 6 and $12 \mathrm{~h}$ ). The cleavage of caspase-3 was
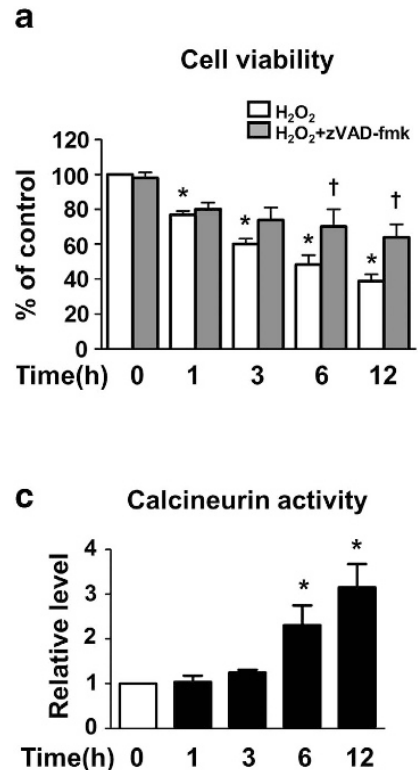

b

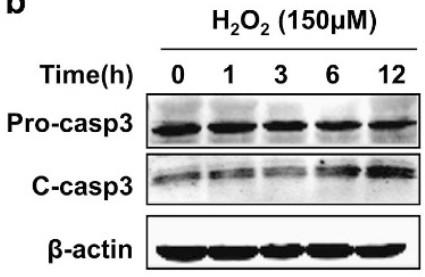

d

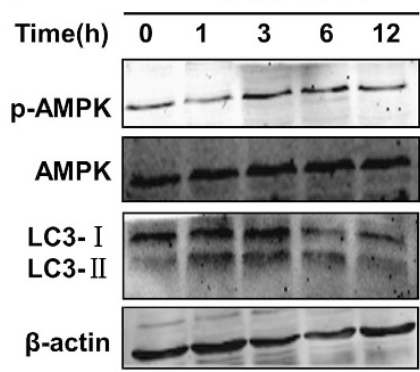

C-casp3
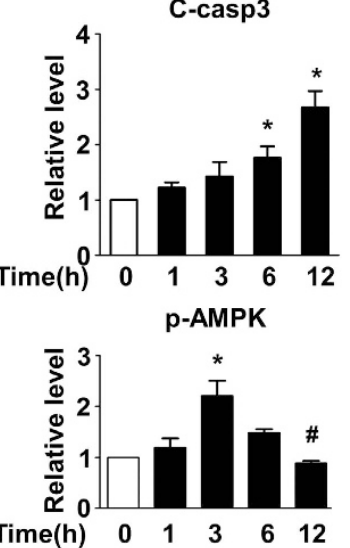

LC3-II

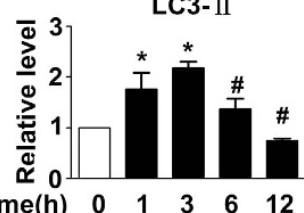

Figure 1 The effect of hydrogen peroxide $\left(\mathrm{H}_{2} \mathrm{O}_{2}\right)$ on cardiomyocyte apoptosis, calcineurin activity, AMPK phosphorylation and LC3 expression. Primary cardiomyocytes were treated with $\mathrm{H}_{2} \mathrm{O}_{2}$ at a final concentration of $150 \mu \mathrm{M}$ for $1,3,6$ or $12 \mathrm{~h}$. (a) The effect of $\mathrm{H}_{2} \mathrm{O}_{2}$ on cell viability at various time points with or without $\mathrm{zVAD}$-fmk. (b) $\mathrm{H}_{2} \mathrm{O}_{2}$ induces time-dependent activation of caspase- 3 as shown by western blotting. The statistical bar graph (right panel) shows the comparison of cleaved caspase-3 levels that have been normalised to $\beta$-actin. (c) $\mathrm{H}_{2} \mathrm{O}_{2}$ time dependently enhances calcineurin $(\mathrm{Cn})$ activity. Cells were treated as described. Calcineurin activity in each group was normalised to the control group. (d) The effect of $\mathrm{H}_{2} \mathrm{O}_{2}$ on LC3 and AMPK protein expression at each time point as detected by western blotting. The levels of LC3-II and $\mathrm{p}$-AMPK were normalised to $\beta$-actin and are shown in the bar graph (right panel). Data are presented as the mean \pm S.E.M., $n \geq 3$. ${ }^{*} P<0.05$ versus the control group, ${ }^{\#} \mathrm{P}<0.05$ versus $3 \mathrm{~h}$ and ${ }^{\dagger} \mathrm{P}<0.05$ versus $\mathrm{H}_{2} \mathrm{O}_{2}$ alone at 6 and $12 \mathrm{~h}$ 
also used to determine the extent of apoptosis. Consistent with the cell viability assay, $\mathrm{H}_{2} \mathrm{O}_{2}$ treatment increased the cleavage of pro-caspase-3 $(34 \mathrm{kDa})$ to its active form $(17 \mathrm{kDa})$ in a time-dependent manner, as was evident at 6 and $12 \mathrm{~h}$ (Figure 1b).These results suggest that long-term $\mathrm{H}_{2} \mathrm{O}_{2}$ treatment induces caspase-dependent apoptosis in cardiomyocytes.

It has been documented that calcineurin transmits a proapoptotic signal in several apoptosis models. ${ }^{27,28,30-32}$ Therefore, we postulated that a calcineurin-related signal response may be activated because of aberrant calcium signalling under oxidative stress conditions. To test this hypothesis, we performed a cellular calcineurin activity assay. As expected, the calcineurin activity significantly increased by threefold in response to long-term $(12 \mathrm{~h}) \mathrm{H}_{2} \mathrm{O}_{2}$ treatment in a timedependent manner (Figure 1c).

Autophagy can be induced by stressors, including reactive oxygen species (ROS). We next detected the dynamic changes in autophagy by western blot analysis of microtubule-associated protein 1 light chain 3 (LC3), a recognised autophagy marker. Cytosolic LC3-I is converted to LC3-II via lipidation and redistributed to the autophagosome membrane in response to autophagic stimuli. ${ }^{33}$ Thus, LC3-II directly reflects the number of autophagosomes. As shown in Figure $1 \mathrm{~d}, \mathrm{H}_{2} \mathrm{O}_{2}$ dramatically enhanced both LC3-I and LC3-II expression by threefold compared with the control group within the first $3 \mathrm{~h}$ of exposure. Notably, sustained treatment with $\mathrm{H}_{2} \mathrm{O}_{2}$ reduced the levels of both LC3-I and LC3-II.

Autophagy is a dynamic process that involves autophagosome formation and lysosome degradation. To assess the influence of $\mathrm{H}_{2} \mathrm{O}_{2}$ on autophagosome formation and the autophagosome-lysosome fusion process, we transfected cardiomyocytes with a construct expressing monomeric red fluorescent protein/green fluorescent protein (mRFP-GFP) tandem tagged LC3 protein to examine the autophagosome maturation process. In merged images, the yellow and red puncta represent autophagosomes and autolysosomes, respectively, because mRFP but not GFP retains fluorescence in the acidic environment of lysosomes. ${ }^{34}$ Consistent with the results of western blot analysis, we observed that the number of autophagosomes rapidly increased within $3 \mathrm{~h}$ of $\mathrm{H}_{2} \mathrm{O}_{2}$ treatment. Sustained treatment resulted in a decrease in the number of yellow puncta, and the number of red puncta was also slightly reduced (Figure 2 ).

These data indicated that $\mathrm{H}_{2} \mathrm{O}_{2}$ induced autophagic flux; moreover, the number of autophagosomes decreased at the late stage $(6-12 \mathrm{~h})$ of treatment relative to the early stage. A few studies have reported that AMPK plays critical roles in the regulation of autophagosome formation in response to ROS. ${ }^{35,36}$ We evaluated the phosphorylation of AMPK at threonine-172 that directly reflects its enzymatic activity. The changes in the expression of phosphorylated AMPK were similar to those observed for LC3-II. The phosphorylation of AMPK rapidly increased in response to oxidative stress, whereas it declined in a time-dependent manner beyond $3 \mathrm{~h}$. Given these results, we wondered whether the apoptosis induced by prolonged oxidative stress is related to the reduced autophagosome formation and whether calcineurin is implicated in the regulation of autophagy through its effect on the AMPK/mTOR axis.
Inhibition of calcineurin promotes autophagy and alleviates oxidative stress-induced apoptosis in cardiomyocytes. To determine the potential role of calcineurin in autophagy, cardiomyocytes were treated with FK506, a specific calcineurin inhibitor, and subjected to prolonged $\mathrm{H}_{2} \mathrm{O}_{2}$ exposure (12h). Consistent with our original assumption, we observed a pronounced elevation in LC3-II immunoreactivity in the FK506 groups with or without $\mathrm{H}_{2} \mathrm{O}_{2}$ (Figure 3a). Inhibition of calcineurin not only resulted in an increase in the number of autophagosomes, but also enhanced the autophagic flux, as shown by the dramatic

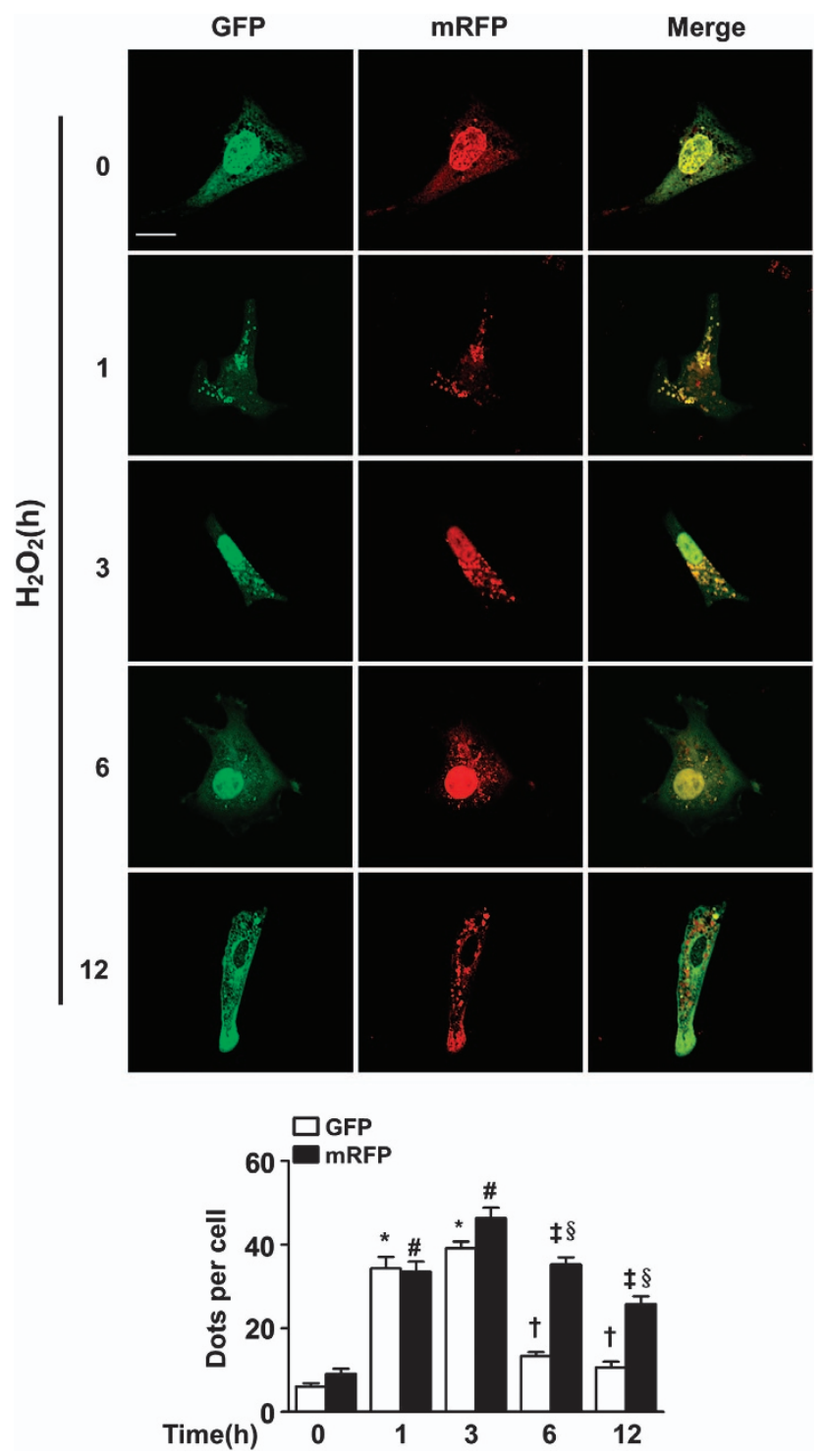

Figure 2 Hydrogen peroxide $\left(\mathrm{H}_{2} \mathrm{O}_{2}\right)$ promotes autophagic flux in cardiomyocytes. Cardiomyocytes were transfected with tandem-LC3 construct (GFP-mRFPLC3) for $24 \mathrm{~h}$, exposed to $150 \mu \mathrm{M} \mathrm{H}_{2} \mathrm{O}_{2}$ for $1,3,6$ or $12 \mathrm{~h}$ and photographed using confocal microscopy. Representative images are shown in the upper panel. Both the GFP and mRFP dots were counted, and the statistical analyses are shown in the bottom panel. The data were obtained from 3 independent experiments, and 10 cells were scored in each experiment. Scale bar $=20 \mu \mathrm{M}$. Data are presented as the mean \pm S.E.M. ${ }^{*} P<0.05$ versus control GFP, ${ }^{\#} P<0.05$ versus control mRFP, ${ }^{\dagger} P<0.05$ versus $3 \mathrm{~h}$ GFP, ${ }^{\ddagger} P<0.05$ versus $3 \mathrm{~h}$ mRFP, and ${ }^{\S} P<0.05$ versus the corresponding GFP dots at each time point 

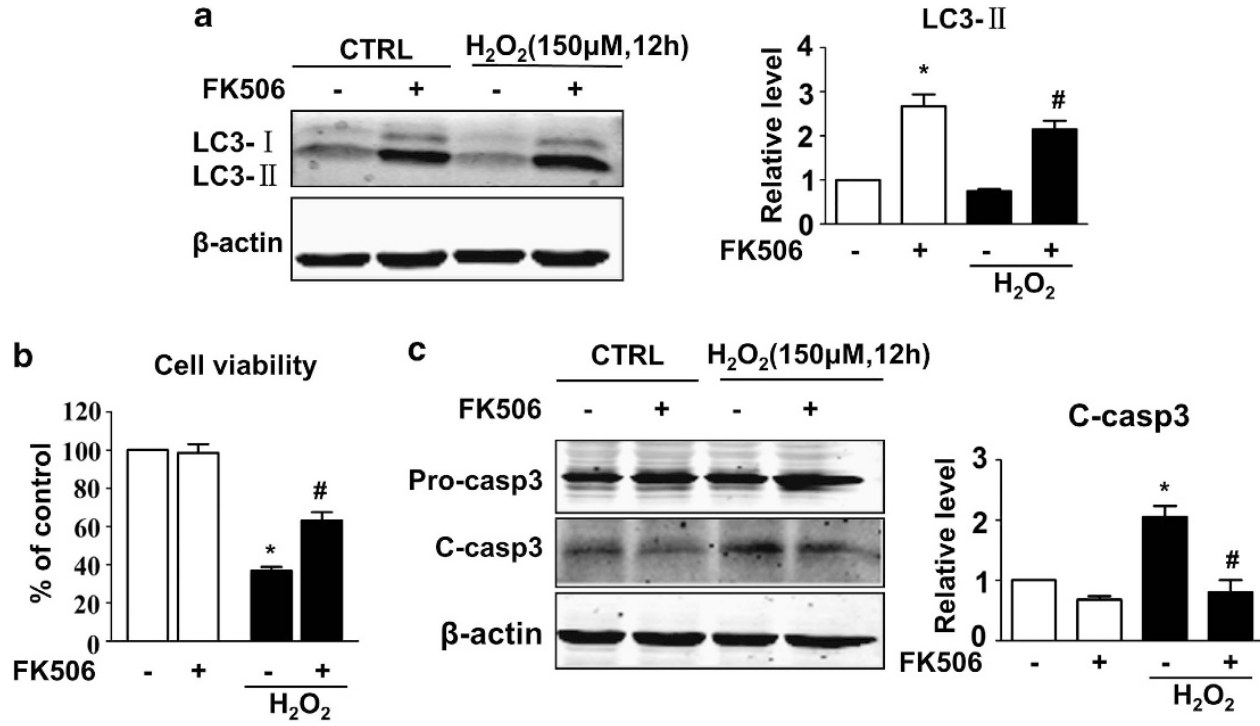

Figure 3 FK506 induces autophagy and alleviates hydrogen peroxide $\left(\mathrm{H}_{2} \mathrm{O}_{2}\right)$-induced apoptosis. Cells were incubated with or without $\mathrm{H}_{2} \mathrm{O}_{2}(150 \mu \mathrm{M})$ in the presence or absence of FK506 $(1 \mu \mathrm{M})$ for $12 \mathrm{~h}$ and subjected to MTT assay and western blotting, respectively. (a) FK506 induced LC3 expression with or without $\mathrm{H}_{2} \mathrm{O}_{2}$. LC3-II levels were normalised to $\beta$-actin and are shown in the bar graph on the right panel. (b) FK506 increased cell viability and (c) decreased the cleavage of caspase-3. The cleaved caspase3 expression was normalised to $\beta$-actin and is shown in the right panel. Data are presented as the mean \pm S.E.M., $n \geq 3 .{ }^{*} P<0.05$ versus the control group and ${ }^{\sharp} P<0.05$ versus the $\mathrm{H}_{2} \mathrm{O}_{2}$ alone group

increase in the number of both GFP and mRFP puncta (Figure 4).

Previous studies have demonstrated that calcineurin induces apoptosis in mammalian cells, including cardiomyocytes. The role of calcineurin in the apoptotic model we used has seldom been tested. We observed that inhibition of calcineurin exerts a protective effect. As shown in Figure $3 a$ and b, cells treated with FK506 displayed decreased cleavage of pro-caspase-3 after long-term (12 h) $\mathrm{H}_{2} \mathrm{O}_{2}$ treatment. The cell viability recovered by an average of $20 \%$ as detected by MTT assay. These results showed that inhibition of calcineurin enhances autophagy and protects cardiomyocytes from oxidative stress-induced apoptosis.

Overexpression of calcineurin inhibits autophagy and exaggerates oxidative stress-induced apoptosis. To further ascertain the inhibitory role of calcineurin on autophagy, cardiomyocytes were transiently transfected with a construct expressing the constitutively active form of calcineurin $A(\mathrm{CnA})$. The cells were subsequently subjected to $\mathrm{H}_{2} \mathrm{O}_{2}$ or control vehicle (phosphate-buffered saline (PBS)) treatment for $3 \mathrm{~h}$, a time point that showed maximum autophagosome formation and mild apoptosis (Figures 1b, $d$ and 2). Overexpression of calcineurin resulted in reduced LC3-II levels with or without $\mathrm{H}_{2} \mathrm{O}_{2}$ (Figure 5a). Moreover, although overexpression of calcineurin A did not markedly change caspase-3 cleavage under normal conditions, we observed increased activation of caspase- 3 and decreased viability of cells after brief exposure to $\mathrm{H}_{2} \mathrm{O}_{2}(3 \mathrm{~h})$ that were comparable to those observed after long-term $\mathrm{H}_{2} \mathrm{O}_{2}$ treatment (12 h) (Figure $5 \mathrm{~b}$ and $\mathrm{c}$ ). These results strongly indicate that calcineurin is a negative regulator of autophagy and exerts a pro-apoptotic effect during oxidative stress.
The AMPK/mTOR axis mediates the effect of calcineurin on autophagy and cell survival during oxidative stress. AMPK/mTOR is critical in the regulation of autophagy, and a positive correlation between phosphorylation of AMPK and LC3-II expression was observed in our cellular model. We thus simultaneously evaluated the changes in the AMPK signalling pathway during both calcineurin inhibition and overexpression. As shown in Figure 6a, inhibition of calcineurin by FK506 significantly restored the phosphorylation of AMPK at $12 \mathrm{~h}$ in response to $\mathrm{H}_{2} \mathrm{O}_{2}$ treatment. Accordingly, the activity of the mTOR signalling, which negatively modulates autophagy and is known to be inhibited by AMPK, was downregulated when treated with FK506, as represented by decreased phosphorylation of mTOR (Ser2448) and its two primary downstream targets p70 ribosomal protein S6 kinase (p70S6K; Thr389) and elongation factor 4E binding protein 1 (4E-BP1; Thr37/46). In contrast, overexpression of the active form of calcineurin attenuated AMPK signalling and enhanced mTOR signalling under both normal and oxidative stress conditions (Figure 6b).

Because calcineurin has been shown to dephosphorylate protein kinase $B(A k t),{ }^{37}$ which is also a potent regulator of mTOR, we examined whether Akt is involved in the regulation of mTOR by calcineurin. As shown in Figure $6 \mathrm{c}$, inhibition of calcineurin by FK506 significantly increased the phosphorylation of Akt under normal conditions. However, this effect was not observed after $\mathrm{H}_{2} \mathrm{O}_{2}$ treatment. Similarly, calcineurin overexpression resulted in a significant decrease in the phosphorylation of Akt that was not observed with $\mathrm{H}_{2} \mathrm{O}_{2}$. These results indicate that $\mathrm{Akt}$ is not involved in the regulation of mTOR by calcineurin under oxidative stress conditions.

To further address the mechanisms by which calcineurin inhibits autophagy, we manipulated AMPK activity by 


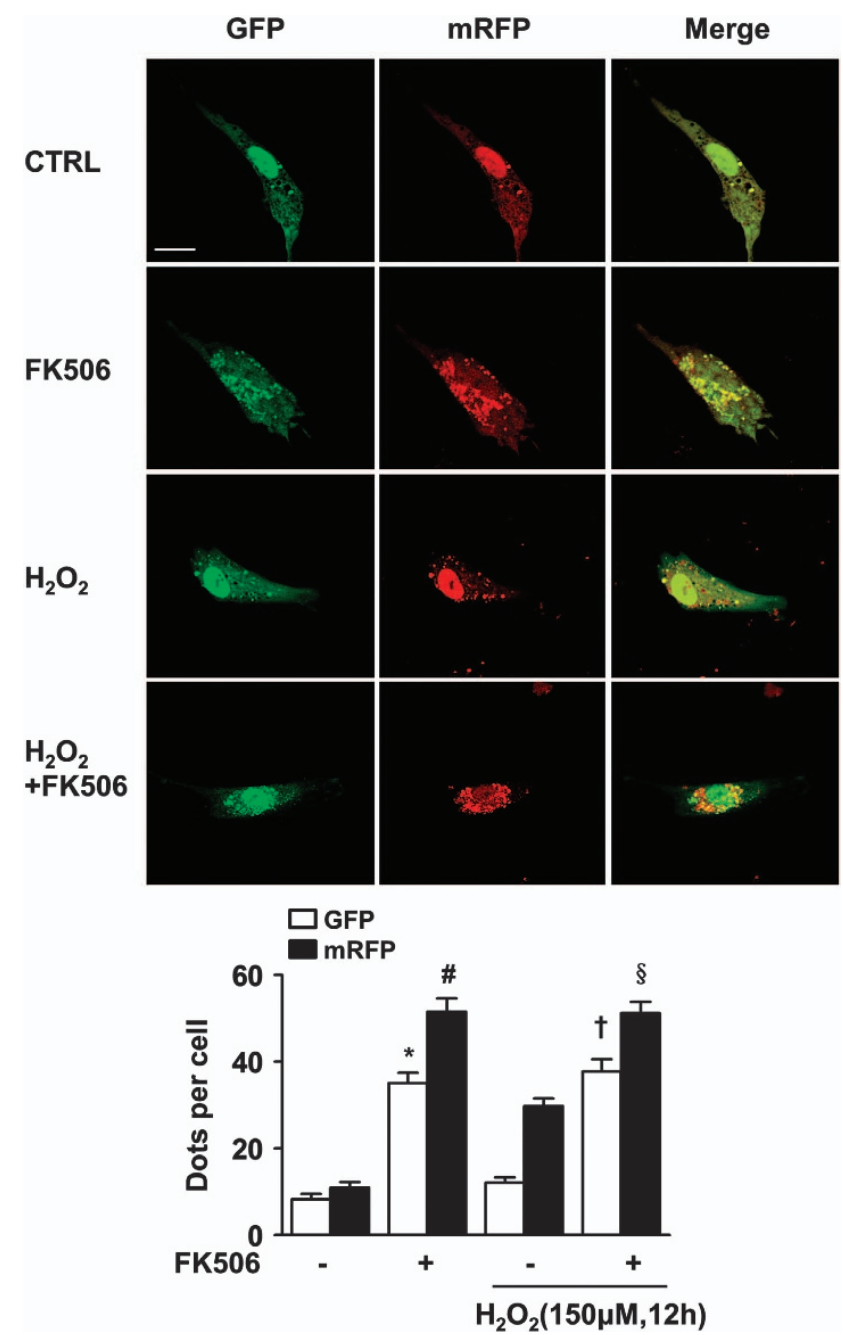

Figure 4 FK506 promotes the formation of autophagosomes and autolysosomes. Cells were transfected as described in Figure 2 and were subsequently treated as described in Figure 3 . The number of both the GFP and mRFP dots was significantly increased with or without $\mathrm{H}_{2} \mathrm{O}_{2}$ treatment as shown in the bar graph in the bottom panel. Scale bar $=20 \mu \mathrm{M}$. Data are presented as the mean \pm S.E.M. ${ }^{*} P<0.05$ versus control GFP, ${ }^{\#} P<0.05$ versus control mRFP, ${ }^{\dagger} P<0.05$ versus $\mathrm{H}_{2} \mathrm{O}_{2}$-alone GFP and ${ }^{\S} \mathrm{P}<0.05$ versus $\mathrm{H}_{2} \mathrm{O}_{2}$-alone mRFP

expressing a dominant negative mutant of AMPK (DN-AMPK), thereby inhibiting endogenous AMPK activity. Cells expressing the kinase-dead K45R DN-AMPK $\alpha 2$ mutant exhibit greater basal phosphorylation levels as previously described. ${ }^{38}$ As shown in Figure 6d, treatment with FK506 resulted in increased levels of LC3-II in cells expressing the control vector, whereas this effect was abolished in cells expressing DN-AMPK as shown by the unchanged LC3-II levels. FK506 failed to blunt mTOR signalling in cells expressing the DN-AMPK mutant. The activation of caspase-3 was also analysed by western blotting. As shown in Figure 6d, inhibition of calcineurin by FK506 decreased the level of cleaved caspase- 3 in cells transfected with the control vector, whereas this effect was abolished in cells expressing the DN-AMPK mutant. These results indicate that the AMPK/ mTOR axis is responsible for the induction of autophagy caused by calcineurin inhibition and to a certain extent mediates a protective effect against oxidative damage.
Calcineurin induces apoptosis through suppression of autophagy during oxidative stress. To investigate the role of autophagy in cell survival under oxidative stress conditions, we manipulated autophagy activity using rapamycin and 3-methyladenine (3-MA) to inhibit the activity of mTOR and class III PI3K, respectively, and to promote or suppress autophagy, respectively. As expected, western blotting and TUNEL (terminal deoxynucleotidyl transferase dUTP nickend labelling) assays both showed that rapamycin attenuated the activation of caspase-3 and reduced the number of TUNEL-positive cells while inducing autophagy. FK506 mimicked the effects of rapamycin; however, 3-MA cancelled the protective effect of FK506 (Figure 7a and b).

In view of the fact that 3-MA and rapamycin have pleiotropic effects besides autophagy modulation, we synthesised small interfering RNA (siRNA) against essential autophagy gene ATG7 to inhibit autophagy more specifically. Knockdown of ATG7 significantly inhibited the autophagy marker LC3-II (Figure 7c). Consistent with the results obtained from pharmacological modulation of autophagy, gene-specific inhibition of autophagy by ATG7 knockdown not only increased $\mathrm{H}_{2} \mathrm{O}_{2}$-induced apoptosis but also markedly blocked the protective effects of calcineurin inhibitor FK506 (Figure 7c and $d$ ). These results confirmed our hypothesis that autophagy is an adaptive response that protects cardiomyocytes under oxidative stress from apoptosis and that increased autophagic activity at least partially confers the protective effects of calcineurin inhibition.

\section{Discussion}

In this study, we demonstrated that calcineurin confers oxidative stress damage on cardiomyocytes by negatively regulating cardiomyocyte autophagy through the AMPK/ mTOR axis. The following evidence supports this conclusion: (1) inhibition of calcineurin dramatically enhanced AMPK/ mTOR signalling, increased autophagy and partially rescued cardiomyocytes from cell death; (2) overexpression of calcineurin attenuated AMPK/mTOR signalling, decreased autophagy and exacerbated oxidative stress damage; (3) the induction of autophagy caused by calcineurin inhibition was abolished in cardiomyocytes expressing dominant negative mutant AMPK; and (4) inhibition of autophagy by 3-MA and ATG7 knockdown abrogated the protective effects of FK506.

In the case of oxidative stress, interaction of ROS and $\mathrm{Ca}^{2+}$ as inducer and effector alters several signalling pathways that are critical to cell survival. As a calcium-activated phosphatase, calcineurin is abundantly expressed in the heart and plays pivotal roles in many cardiovascular diseases involving oxidative stress. ${ }^{26,39,40}$ It is still unknown whether calcineurin delivers pro-survival or pro-death signals in the heart. Transgenic mice that constitutively activate calcineurin A exhibit less apoptosis than WT littermates. ${ }^{41}$ In contrast, calcineurin induces apoptosis through dephosphorylation of ASK1 and ARC in cardiomyocytes exposed to $\mathrm{H}_{2} \mathrm{O}_{2}$ and isoprenaline, respectively. ${ }^{27,30} \mathrm{~A}$ more recent study revealed a novel role of calcineurin in transmitting the apoptotic signal during heart ischaemia-reperfusion by regulating mitochondrial dynamics. ${ }^{28}$ Although it is well known that calcineurin directly triggers apoptosis or mediates the pro-apoptotic effect 

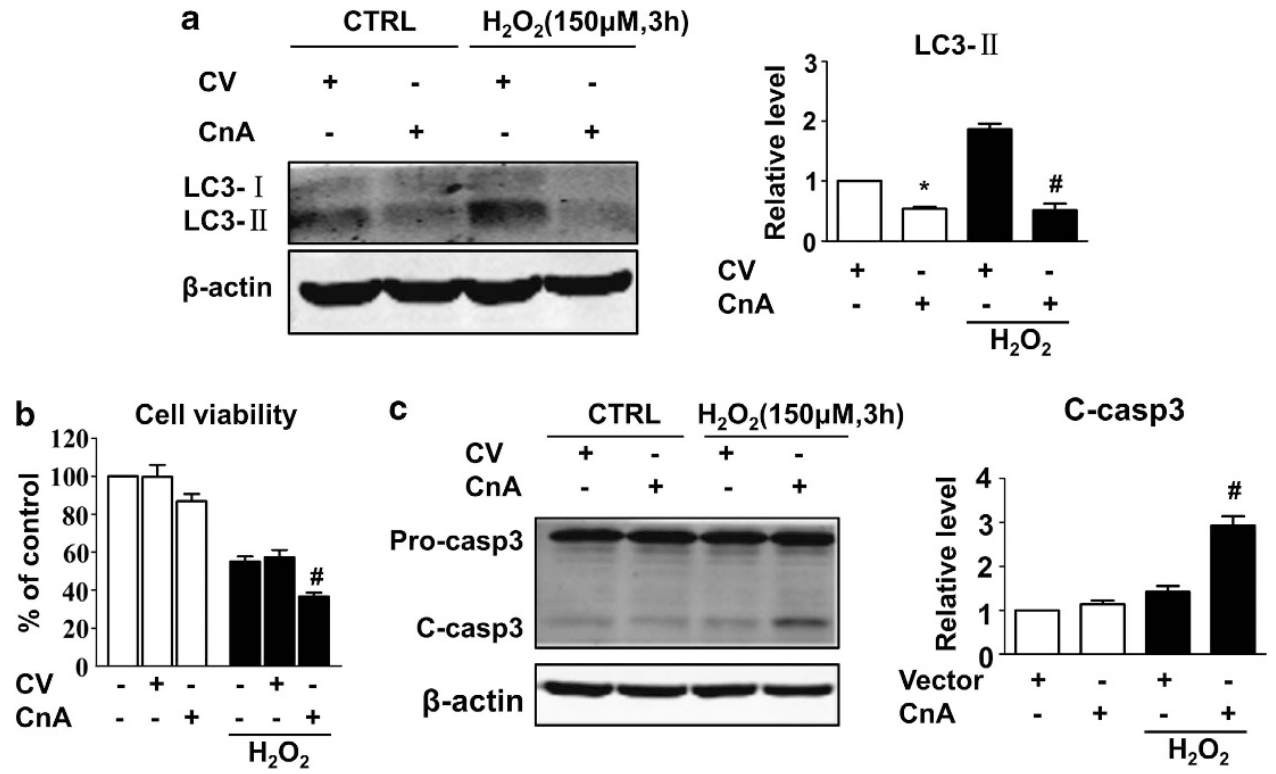

Figure 5 Overexpression of calcineurin inhibits autophagy and sensitises cells to undergo $\mathrm{H}_{2} \mathrm{O}_{2}$-induced apoptosis. Cells were transfected with the control vector (CV) or vector expressing calcineurin A for $24 \mathrm{~h}$ and subsequently incubated with or without $\mathrm{H}_{2} \mathrm{O}_{2}(150 \mu \mathrm{M})$ for $3 \mathrm{~h}$. After the treatment, the cells were harvested for MTT assay and western blotting. (a) Calcineurin inhibits LC3 expression whether $\mathrm{H}_{2} \mathrm{O}_{2}$ is present or not. (b) Overexpression of calcineurin results in decreased cell viability and (c) increased caspase-3 cleavage. Data are presented as the mean \pm S.E.M., $n \geq 3 .{ }^{*} P<0.05$ versus the control group and ${ }^{\#} P<0.05$ versus the $\mathrm{H}_{2} \mathrm{O}_{2}$-alone group

in cardiomyocytes and other cells, the link between calcineurin, autophagy and apoptosis remains obscure. Our present study suggests for the first time that apoptosis is induced at least partially by the retardation of autophagy caused by calcineurin activation. Consistent with other studies, ${ }^{9,42,43}$ we demonstrated that AMPK/mTOR-dependent autophagy is a mechanism that protects against oxidative stress.

Excessive ROS has the potential to cause protein misfolding and mitochondrial damage $\mathrm{e}^{44,45}$ that are detrimental to proper mitochondrial function and cause energy and nutrient depletion. Autophagy is evolutionarily utilised by eukaryotic cells to recycle cellular waste into raw materials and thereby maintain homeostasis. ${ }^{5,6}$ Given that a cardiomyocyte is a nonreplicating cell with a high metabolism, it is of great importance for cardiomyocytes to maintain high autophagic activity in response to oxidative stress. Because ROS cause various types of cell damage, such as energy deprivation, DNA damage, calcium abnormalities and mitochondrial aggregation, autophagy may be induced by perturbations to a series of signals. The role of AMPK, which senses energy status and regulates autophagy, has been well established. AMPK is able to induce autophagy through direct activation of Ulk1 (mammalian homologue of ATG1 in yeast) ${ }^{19}$ and inhibition of mTORC1. ${ }^{46,47}$ Given that calcium is one of the most common and effective signal transducers and plays key roles in oxidative stress, ${ }^{48}$ calcium signals may tune autophagy. Recent studies implicate calcium signalling in the induction of autophagy via calcium/calmodulin-dependent protein kinase kinase $\beta$ (CaMKK $\beta$ )-dependent activation of AMPK. ${ }^{49}$ In our study, however, we demonstrated that the calcineurin signal suppresses autophagy by counteracting the AMPK/mTOR axis that is consistent with previous studies in calcineurin-null mutants of $C$. elegans and in the cyclosporine A-treated rat hippocampus. ${ }^{29,50}$
In our study, autophagosomes were rapidly assembled at an early stage after oxidative stress stimulus, but their numbers declined remarkably after continued stimulation. By utilising the tandem fluorescence LC3 microscopy assay, we verified the autophagic flux induced by oxidative stress. It is worth noting that the attenuation of the AMPK signal at late stages was observed in our experiments. Because AMPK functions as a potent autophagy regulator that affects autophagosome formation through a mTOR- and Ulk1-dependent mechanism, we still cannot rule out the contributions of attenuated AMPK signalling to the decrease in the number of autophagosomes at the late stage relative to the early stage, even though the decrease can be explained by the degradation of LC3 by the autophagosome clearance mechanism when it fuses with the lysosome. By either inhibiting or overexpressing calcineurin, we discovered that calcineurin regulates autophagy through the AMPK pathway. We have unveiled the role of AMPK-mediated autophagy on cell survival. Collectively, we proposed the following schematic model for the role of calcineurin in the regulation of oxidative stress damage. At early stages, the adaptive response is triggered and leads to AMPK activation, causing a higher autophagy level to maintain homeostasis. Despite modest increases in calcineurin activity, the adaptive signals activating AMPK are strong enough to offset the antagonistic effect of calcineurin on AMPK. However, with long-term exposure, a sustained increase in calcineurin activity beyond a certain threshold is sufficient to inhibit the AMPK pathway, thereby inhibiting adaptive autophagy with resultant cell death.

Although we identified AMPK as a main mediator of autophagy that is controlled by calcineurin, we did not exclude other mechanisms. To present studies, mTOR is at the convergence of multiple signals that modulate autophagy and is precisely regulated by upstream kinase cascades. Stressed cells often exhibit alternations of several different signals 
a

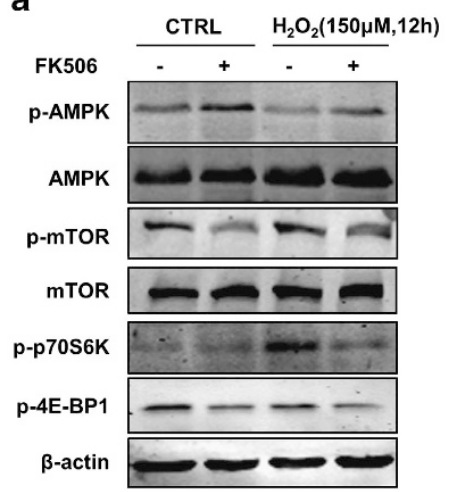

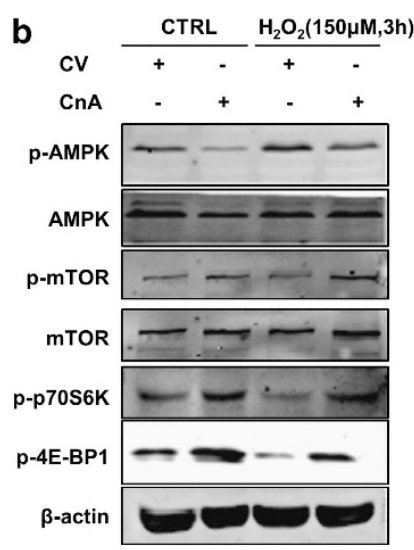
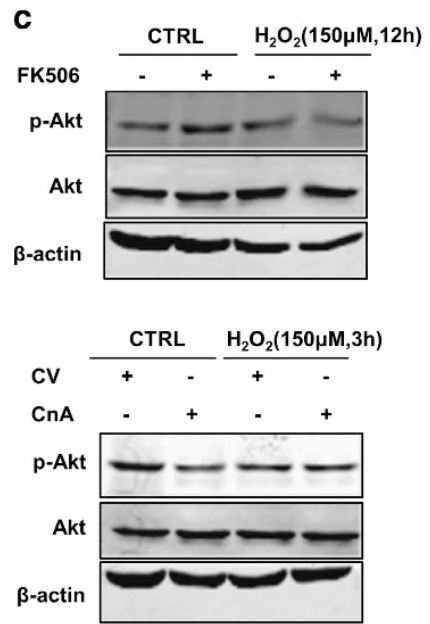

d

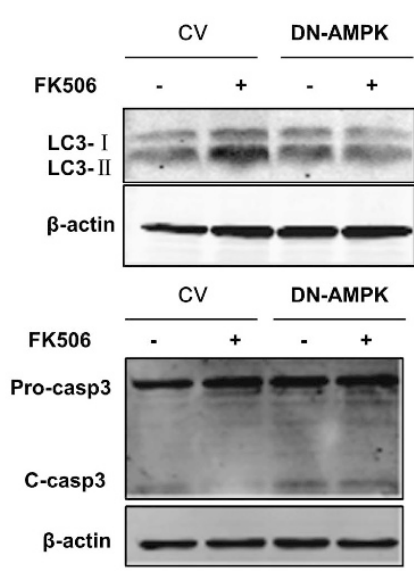

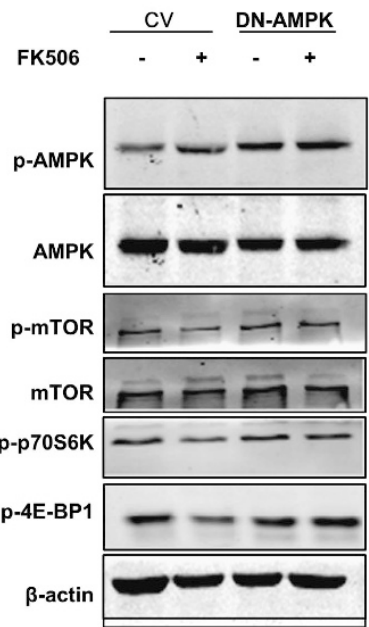

Figure 6 AMPK/mTOR signalling mediates the effect of calcineurin on autophagy and cell survival after $\mathrm{H}_{2} \mathrm{O}_{2}$ treatment. (a) Inhibition of calcineurin by FK506 enhances AMPK phosphorylation and attenuates the phosphorylation of mTOR. Cells were treated as described in Figure 3 , and whole-cell lysates were subjected to western blot analysis. (b) Overexpression of calcineurin attenuates the phosphorylation of AMPK and enhances mTOR phosphorylation. Cells were treated as described in Figure 5, and whole-cell lysates were subjected to western blot analysis. (c) Calcineurin attenuates Akt signalling under normal conditions but not under oxidative stress conditions. Cells were treated as previously described. (d) FK506 showed no effect on autophagy regulation, cell protection and mTOR inhibition in DN-AMPK-expressing cells. Cells were transfected with the control vector (CV) and the DN-AMPK vector for $24 \mathrm{~h}$ and subsequently incubated with $150 \mu \mathrm{M} \mathrm{H}_{2} \mathrm{O}_{2}$ in the presence or absence of FK506 (1 $\left.\mu \mathrm{M}\right)$ for $12 \mathrm{~h}$. Representative images are from three or more independent experiments

concurrently. Oxidative stress may affect mTOR at different levels. For example, the PI3K/Akt/mTOR axis is extensively studied in the regulation of autophagy. PI3K/Akt signalling negatively regulates autophagy by activating mTOR. ${ }^{51,52}$ Calcineurin has been reported to directly dephosphorylate Akt. ${ }^{37}$ Indeed, our results are in agreement with this study when cells are under normal conditions. However, we did not observe that calcineurin has a significant effect on Akt signalling in the context of oxidative stress. Inhibition of AMPK by overexpressing the DN-AMPK construct completely abolished the effect of FK506 on mTOR and the outcome of autophagy. These results strongly suggest that calcineurin regulates $\mathrm{mTOR}$ in an AMPK-dependent, Akt-independent manner. In addition to regulation through the AMPK/mTOR axis, calcineurin might affect autophagy by transcriptional modulation. Recent studies have shed some light on the relationship between calcineurin and forkhead transcriptional factors (FOXO). ${ }^{53,54} \mathrm{FOXO} 1$ and $\mathrm{FOXO} 3$ are able to regulate autophagy-related gene expression in cardiomyocytes. ${ }^{55}$ Whether calcineurin regulation of cardiomyocyte autophagy involves a FOXO-dependent mechanism under oxidative stress conditions remains to be seen.

Despite that we have found that calcineurin attenuates AMPK-dependent autophagy, our understanding of how AMPK is regulated by calcineurin is still rudimentary. AMPK activity is precisely controlled by kinases that lead to phosphorylation at the Thr-172 residue. We speculate that calcineurin attenuates AMPK signalling through indirect regulation, because the dephosphorylation of AMPK is primarily under the control of PP2C $\alpha$ and PP2A. ${ }^{56,57}$ TGF$\beta$-activated kinase 1 (TAK1), liver kinase B1 (LKB1) and CaMKK $\beta$ are upstream kinases of AMPK. In a study of the rat hippocampus, ${ }^{29}$ cyclosporine $A$ enhanced the phosphorylation of activation residues of TAK1 and LKB1. As a protein phosphatase, calcineurin may have the potential to dephosphorylate these kinases under calcium perturbation conditions. Although TAK1 has been identified as a target of calcineurin, ${ }^{58}$ it will be interesting in our future work to determine whether calcineurin dephosphorylates these three kinases in this cellular model. 
a

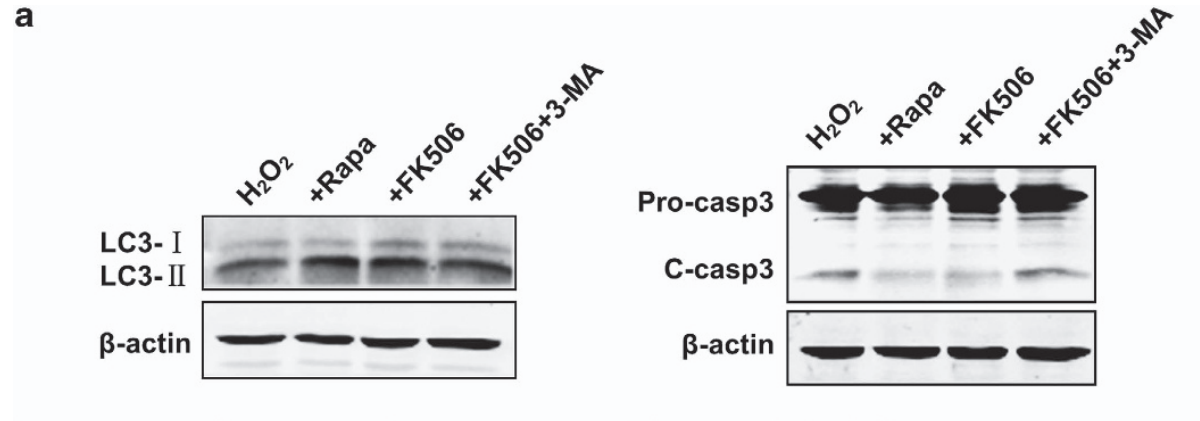

b
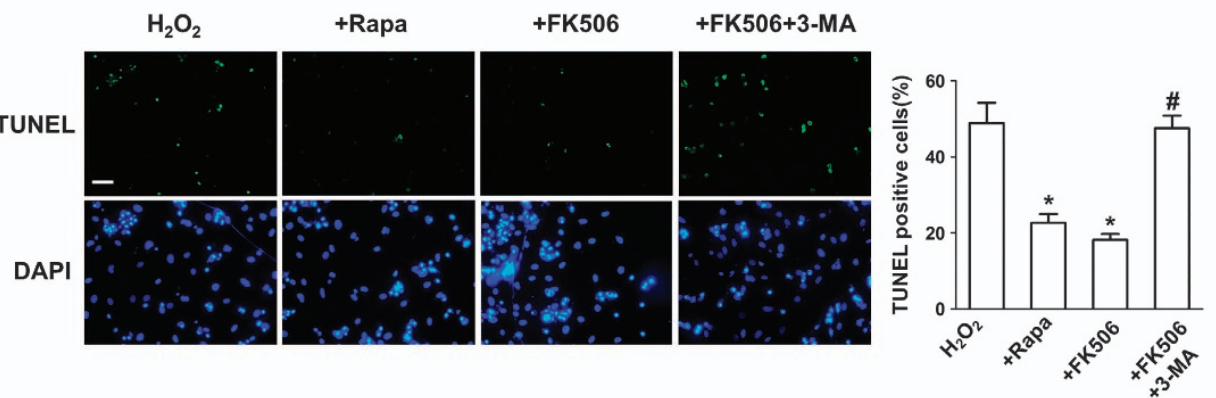

C
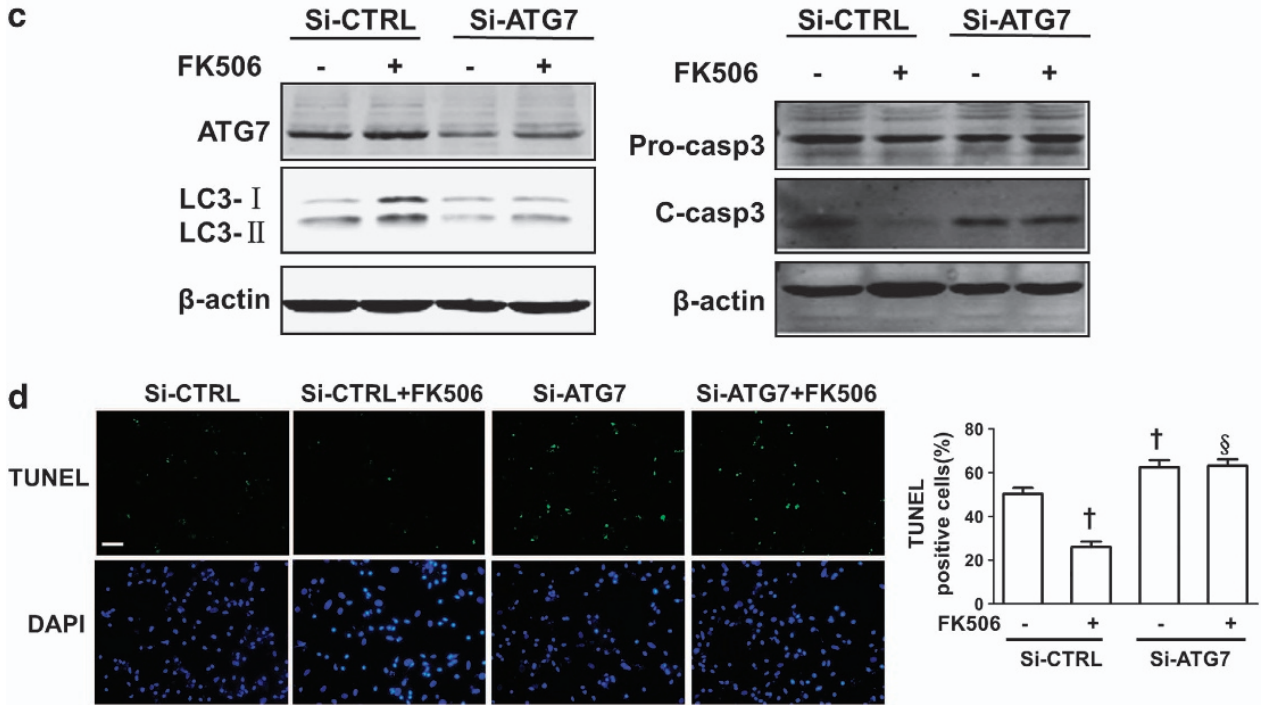

Figure 7 Autophagy protects cells from apoptosis induced by hydrogen peroxide $\left(\mathrm{H}_{2} \mathrm{O}_{2}\right)$. (a) The effect of rapamycin, FK506 and FK506 combined with 3-MA on LC3-II and cleaved caspase-3 expression as detected by western blotting. (b) The effect of rapamycin, FK506 and FK506 combined with 3-MA on H2O2-induced apoptosis as determined by TUNEL staining. Cells were incubated with $\mathrm{H}_{2} \mathrm{O}_{2}(150 \mu \mathrm{M})$ in the presence of rapamycin (Rapa, $\left.500 \mathrm{nM}\right)$, FK506 $(1 \mu \mathrm{M})$ or FK506 $(1 \mu \mathrm{M})$ combined with 3-MA $(5 \mathrm{mM})$ for $12 \mathrm{~h}$. (c) The effect of ATG7 knockdown on LC3-II and cleaved caspase-3 expression detected by western blotting. (d) The effect of ATG7 knockdown on $\mathrm{H}_{2} \mathrm{O}_{2}$ induced apoptosis as determined by TUNEL staining. Cells were transfected with negative control or ATG7 siRNA for $48 \mathrm{~h}$ and were subsequently subjected to $\mathrm{H}_{2} \mathrm{O}_{2}(150 \mu \mathrm{M})$ for $12 \mathrm{~h}$ with or without FK506 $(1 \mu \mathrm{M})$. Scale bar $=100 \mu \mathrm{M}$, the data for statistical analysis were from three independent experiments and the percentage of TUNEL-positive cells was scored in four non-overlapping microscopic fields in each experiment. Data are presented as the mean \pm S.E.M. ${ }^{*} P<0.05$ versus $\mathrm{H}_{2} \mathrm{O}_{2}$ alone and ${ }^{\#} P<0.05$ versus $\mathrm{H}_{2} \mathrm{O}_{2}+\mathrm{FK} 506,{ }^{\dagger} P<0.05$ versus si-CTRL and ${ }^{\S} P<0.05$ versus si-CTRL + FK506

The current study is important because our findings define a novel interaction between calcineurin and the AMPK/ mTOR pathway for the control of autophagy. We demonstrated that autophagy is a critical biologic process for the survival of cardiomyocytes undergoing oxidative stress. Because oxidative stress is involved in a series of cardiovascular complications, inhibition of calcineurin and activation of autophagy may be a novel therapeutic approach with a broad clinical potential to prevent oxidative stress damage to cardiomyocytes.

\section{Materials and Methods}

Cell culture and treatment. Primary rat cardiomyocytes were prepared as previously described. ${ }^{59}$ Briefly, cardiac tissues were digested by pancreatin, and the isolated cells were resuspended in Dulbecco's modified Eagle's medium (DMEM; Hyclone, Logan, UT, USA) containing 10\% fetal bovine serum FBS; Hyclone). Cardiomyocytes were purified by differential plating, and $0.1 \mathrm{mmol} / \mathrm{l}$ BrdU (5-bromo-2-deoxyuridine) was added to exclude the cardiac fibroblasts. Cells were cultured in a $5 \% \mathrm{CO}_{2}$ and $37^{\circ} \mathrm{C}$ humidified atmosphere, and subsequent experiments were performed $48 \mathrm{~h}$ after plating. To simulate oxidative stress in cardiomyocytes, $\mathrm{H}_{2} \mathrm{O}_{2}$ was used at a final concentration of $150 \mu \mathrm{M}$. ZVAD-fmk $(40 \mu \mathrm{M})$, FK506 $(1 \mu \mathrm{M})$ and rapamycin $(500 \mathrm{nM})$ were dissolved in dimethyl 
sulphoxide (DMSO), and 3-MA (5 mM) was dissolved in the culture medium. All the chemical reagents were purchased from Sigma-Aldrich (St. Louis, MO, USA).

Cell viability assay. An MTT assay was used to determine cell viability. Adherent cardiomyocytes were washed and placed in fresh medium. The cells were treated as designated and were subsequently incubated with $20 \mu$ MTT $(0.5 \mathrm{mg} / \mathrm{ml})$ for $4 \mathrm{~h}$. The culture medium was carefully removed, and $200 \mu \mathrm{l}$ DMSO was added to each well to dissolve the formazan. After the cells were rocked for $10 \mathrm{~min}$, the absorbance values were read at $570 \mathrm{~nm}$ using an Infinite m200pro microplate spectrophotometer (Tecan, Salzburg, Austria).

Cellular calcineurin activity assay. Cellular calcineurin activity was measured by calcineurin cellular assay kit (ENZO Life Sciences, Plymouth Meeting, PA, USA) according to the manufacturer's protocol. Briefly, treated cells were collected using the lysis buffer supplied in the kit. The extracts were then subjected to desalting resin to remove free phosphate. The desalted lysates were incubated with the calcineurin substrate RII phosphopeptide in assay buffer and EGTA buffer at $30^{\circ} \mathrm{C}$ for 30 min to determine total phosphatase activity and phosphatase activity without calcineurin, respectively. Then, $100 \mu \mathrm{l} \mathrm{BIOMOL}$ GREEN was added to each well to visualise the phosphate release. The colour was allowed to develop at room temperature for $20 \mathrm{~min}$ before the detection of the absorbance value at $620 \mathrm{~nm}$ on the microplate spectrophotometer. The absorbance value $A_{620}$, total $-A_{620}$, EGTA buffer was converted to phosphate release using the standard curve that was prepared in each assay. The phosphate release was divided by protein concentration and normalised to the control group to obtain relative calcineurin activity.

TUNEL assay. Cells were plated on coverslips in 24-well culture plates and treated as described. To evaluate the extent of apoptosis, a TUNEL assay was performed using an in situ cell death detection kit (Roche, Mannheim, Germany) according to the manufacturer's protocol. The TUNEL-stained slides were washed with PBS and counterstained with 4',6-diamidino-2-phenylindole (DAPI; Beyotime, Shanghai, China). A laser confocal microscope (Olympus, Tokyo, Japan) was used to acquire the images. Nuclei that were double labelled with TUNEL and DAPI were considered to be TUNEL positive. The TUNEL-positive cells were counted in four non-overlapping microscopic fields of three independent experiments.

RNA interference. The siRNA targeting rat ATG7 and nontargeting negative control siRNA were designed and synthesised by Invitrogen (Shanghai, China). The siRNAs were transfected into cells using the Xtreme GENE siRNA Transfection Reagent (Roche) at a concentration of $100 \mathrm{nM}$ according to the manufacturer's instructions. Subsequent experiments were performed $48 \mathrm{~h}$ after the transfection.

Plasmid transfections. The tandem tagged GFP-mRFP-LC3 plasmid was generously provided by Dr. Tamotsu Yoshimori (Addgene plasmid, 21074, Addgene, Cambridge, MA, USA), the pcDNA3.0 plasmid that encodes DNAMPK $\alpha 2$ (K45R) was generously provided by Dr. Morris J Birnbaum (Addgene plasmid, 15992, Addgene) and the pEGFP-C3 plasmid that encodes the constitutively active form of $\mathrm{CnA}$ was generously provided by Dr. Oliver Ritter (Würzburg, Germany). The control vectors pEGFP-C3 and pcDNA3.0 were obtained from Clontech (Palo Alto, CA, USA) and Invitrogen (San Diego, CA, USA) respectively. Primary cardiomyocytes at $70-80 \%$ confluence were transfected with 1.0-2.0 $\mu \mathrm{g}$ plasmid using Xtreme GENE HP (Roche) according to the manufacturer's instructions. The cells were subjected to the different designated treatments after $36 \mathrm{~h}$ of transfection.

Tandem mRFP-GFP fluorescence microscopy. Cells transiently expressing GFP-mRFP-LC3 were treated as designated and were then observed by laser microscopy. The number of GFP and mRFP puncta per cell was quantified manually. At least 10 cells in 3 independent experiments were analysed randomly.

Western blotting. After the treatment described above, cells were lysed in RIPA buffer. The protein concentration was determined with a BCA kit (Beyotime) according to the manufacturer's instructions. For western blot analysis, $20-40 \mu \mathrm{g}$ denatured protein was separated on SDS-PAGE gels and transferred onto nitrocellulose membranes. Before incubation with the primary antibody, the membranes were blocked with $5 \%$ skim milk in TBS for $1 \mathrm{~h}$ at room temperature on a rocker. The membranes were incubated with diluted antibody in TBS containing $5 \% \mathrm{BSA}$ and $0.1 \%$ Tween- 20 at $4{ }^{\circ} \mathrm{C}$ overnight. The membranes were washed 3 times with TBST (TBS containing $0.5 \%$ Tween-20) for 5 min each wash, followed by re-probing with fluorescence-conjugated secondary antibodies at room temperature for $1 \mathrm{~h}$. Membranes were washed again 3 times with TBST for $5 \mathrm{~min}$ each before antibody detection using the Odyssey infrared scanning system (LI-COR Biosciences, Lincoln, NE, USA). The western blot bands were quantified using Odyssey 3.0 software and normalised with respect to the loading control. The antibody sources and dilutions used are as follows:

Antibodies against LC3B $(2775,1: 1000)$, p-AMPK $\alpha$ (2535, 1:500), AMPK $\alpha$ (2531, 1:1000), p-mTOR (5536, 1:1000), mTOR (2983, 1:1000), p-p70S6K (9208, 1:500), p-4EBP1 (2855, 1:1000), caspase-3 (9662, 1:1000), Akt (9272, $1: 1000)$, and ATG7 $(8558,1: 1000)$ were purchased from Cell Signaling Technology (Danvers, MA, USA).

The antibody against p-Akt (sc-7985-R, 1:200) was purchased from Santa Cruz Biotechnology (Santa Cruz, CA, USA).

The antibody against $\beta$-actin (ab8226, 1:2000) was purchased from Abcam (Cambridge, MA, USA).

The fluorescence-conjugated secondary IRDye700/800 mouse and rabbit antibodies $(1: 10000)$ were purchased from LI-COR.

Statistical analysis. Quantitative data were reported as the mean \pm S.E.M., and one-way analysis of variance (ANOVA) was used to examine the difference between groups. Pairwise comparisons were performed using the Bonferroni post hoc test. A two-tailed value of $P<0.05$ was considered statistically significant.

\section{Conflict of Interest}

The authors declare no conflict of interest.

Acknowledgements. We thank Professor O Ritter and Dr. Melanie Mühlfelder (Würzburg, Germany) for providing the calcineurin plasmid. This work was supported in part by National Basic Research Program of China (973 Program) (2013CB531104), the Major Program (81230081) of National Natural Science Foundation of China and the National Nature Science Foundation of China (No. 31171094 and 81100122).

1. Dhalla NS, Temsah RM, Netticadan T. Role of oxidative stress in cardiovascular diseases. J Hypertens 2000; 18: 655-673.

2. Ansley DM, Wang B. Oxidative stress and myocardial injury in the diabetic heart. J Pathol 2013; 229: 232-241.

3. Kubli DA, Gustafsson AB. Mitochondria and mitophagy: the yin and yang of cell death control. Circ Res 2012; 111: 1208-1221.

4. Kongara S, Karantza V. The interplay between autophagy and ROS in tumorigenesis. Front Oncol 2012; 2: 171.

5. Levine B, Kroemer G. Autophagy in the pathogenesis of disease. Cell 2008; 132: 27-42.

6. Mizushima N, Levine B, Cuervo AM, Klionsky DJ. Autophagy fights disease through cellular self-digestion. Nature 2008; 451: 1069-1075.

7. Kroemer G, Levine B. Autophagic cell death: the story of a misnomer. Nat Rev Mol Cell Biol 2008; 9: 1004-1010.

8. Kuma A, Hatano M, Matsui M, Yamamoto A, Nakaya H, Yoshimori T et al. The role of autophagy during the early neonatal starvation period. Nature 2004; 432: 1032-1036.

9. Dutta D, Xu J, Kim JS, Dunn WA Jr, Leeuwenburgh C. Upregulated autophagy protects cardiomyocytes from oxidative stress-induced toxicity. Autophagy 2013; 9: 328-344.

10. Yin JJ, Li YB, Wang Y, Liu GD, Wang J, Zhu XO et al. The role of autophagy in endoplasmic reticulum stress-induced pancreatic beta cell death. Autophagy 2012; 8: 158-164.

11. Ciechomska IA, Gabrusiewicz K, Szczepankiewicz AA, Kaminska B. Endoplasmic reticulum stress triggers autophagy in malignant glioma cells undergoing cyclosporine a-induced cell death. Oncogene 2013; 32: 1518-1529.

12. Xie Z, Lau K, Eby B, Lozano P, He C, Pennington B et al. Improvement of cardiac functions by chronic metformin treatment is associated with enhanced cardiac autophagy in diabetic OVE26 mice. Diabetes 2011; 60: 1770-1778.

13. Zhu XC, Yu JT, Jiang T, Tan L. Autophagy modulation for Alzheimer's disease therapy. Mol Neurobiol 2013; 48: 702-714

14. Komatsu M, Waguri S, Chiba T, Murata S, Iwata J, Tanida I et al. Loss of autophagy in the central nervous system causes neurodegeneration in mice. Nature 2006; 441: 880-884.

15. Nakai A, Yamaguchi O, Takeda T, Higuchi Y, Hikoso S, Taniike M et al. The role of autophagy in cardiomyocytes in the basal state and in response to hemodynamic stress. Nat Med 2007; 13: 619-624. 
16. Kang HT, Lee KB, Kim SY, Choi HR, Park SC. Autophagy impairment induces premature senescence in primary human fibroblasts. PLoS One 2011; 6: e23367.

17. Hosokawa N, Hara T, Kaizuka T, Kishi C, Takamura A, Miura $Y$ et al. Nutrient-dependent mTORC1 association with the ULK1-Atg13-FIP200 complex required for autophagy. Mol Biol Cell 2009; 20: 1981-1991.

18. Jung $\mathrm{CH}$, Jun $\mathrm{CB}$, Ro SH, Kim YM, Otto NM, Cao J et al. ULK-Atg13-FIP200 complexes mediate mTOR signaling to the autophagy machinery. Mol Biol Cell 2009; 20: 1992-2003.

19. Kim J, Kundu M, Viollet B, Guan KL. AMPK and mTOR regulate autophagy through direct phosphorylation of Ulk1. Nat Cell Biol 2011; 13: 132-141.

20. Alers S, Loffler AS, Wesselborg S, Stork B. Role of AMPK-mTOR-Ulk1/2 in the regulation of autophagy: cross talk, shortcuts, and feedbacks. Mol Cell Biol 2012; 32: 2-11.

21. Hawley SA, Davison M, Woods A, Davies SP, Beri RK, Carling D et al. Characterization of the AMP-activated protein kinase kinase from rat liver and identification of threonine 172 as the major site at which it phosphorylates AMP-activated protein kinase. J Biol Chem 1996; 271: 27879-27887.

22. Heidrich F, Schotola H, Popov AF, Sohns C, Schuenemann J, Friedrich M et al. AMPK - activated protein kinase and its role in energy metabolism of the heart. Curr Cardiol Rev 2010; 6: 337-342.

23. Paiva MA, Rutter-Locher Z, Goncalves LM, Providencia LA, Davidson SM, Yellon DM et al. Enhancing AMPK activation during ischemia protects the diabetic heart against reperfusion injury. Am J Physiol Heart Circ Physiol 2011; 300: H2123-H2134.

24. Kong SS, Liu JJ, Yu XJ, Lu Y, Zang WJ. Protection against ischemia-induced oxidative stress conferred by vagal stimulation in the rat heart: involvement of the AMPK-PKC pathway. Int J Mol Sci 2012; 13: 14311-14325.

25. Russell RR 3rd, Li J, Coven DL, Pypaert M, Zechner C, Palmeri M et al. AMP-activated protein kinase mediates ischemic glucose uptake and prevents postischemic cardiac dysfunction, apoptosis, and injury. J Clin Invest 2004; 114: 495-503.

26. Molkentin JD, Lu JR, Antos CL, Markham B, Richardson J, Robbins J et al. A calcineurindependent transcriptional pathway for cardiac hypertrophy. Cell 1998; 93: 215-228.

27. Tan WQ, Wang JX, Lin ZQ, Li YR, Lin Y, Li PF. Novel cardiac apoptotic pathway: the dephosphorylation of apoptosis repressor with caspase recruitment domain by calcineurin Circulation 2008; 118: 2268-2276.

28. Wang JX, Jiao JQ, Li Q, Long B, Wang K, Liu JP et al. miR-499 regulates mitochondrial dynamics by targeting calcineurin and dynamin-related protein-1. Nat Med 2011; 17: 71-78.

29. Park HG, Yi H, Kim SH, Yu HS, Ahn YM, Lee YH et al. The effect of cyclosporine A on the phosphorylation of the AMPK pathway in the rat hippocampus. Prog Neuropsychopharmacol Biol Psychiatry 2011; 35: 1933-1937

30. Liu Q, Wilkins BJ, Lee YJ, Ichijo $H$, Molkentin JD. Direct interaction and reciprocal regulation between ASK1 and calcineurin-NFAT control cardiomyocyte death and growth. Mol Cell Biol 2006; 26: 3785-3797.

31. Springer JE, Azbill RD, Nottingham SA, Kennedy SE. Calcineurin-mediated BAD dephosphorylation activates the caspase-3 apoptotic cascade in traumatic spinal cord injury. J Neurosci 2000; 20: 7246-7251.

32. Wang L, Chang JH, Paik SY, Tang Y, Eisner W, Spurney RF. Calcineurin (CN) activation promotes apoptosis of glomerular podocytes both in vitro and in vivo. Mol Endocrino/2011; 25: $1376-1386$.

33. Kabeya Y, Mizushima N, Ueno T, Yamamoto A, Kirisako T, Noda T et al. LC3, a mammalian homologue of yeast Apg8p, is localized in autophagosome membranes after processing. EMBO J 2000; 19: 5720-5728.

34. Kimura S, Noda T, Yoshimori T. Dissection of the autophagosome maturation process by a novel reporter protein, tandem fluorescent-tagged LC3. Autophagy 2007; 3: 452-460.

35. Wang Q, Liang B, Shirwany NA, Zou MH. 2-Deoxy-D-glucose treatment of endothelial cells induces autophagy by reactive oxygen species-mediated activation of the AMP-activated protein kinase. PLoS One 2011; 6: e17234.

36. Sid B, Verrax J, Calderon PB. Role of AMPK activation in oxidative cell damage: implications for alcohol-induced liver disease. Biochem Pharmacol 2013; 86: 200-209.

37. Ni YG, Wang N, Cao DJ, Sachan N, Morris DJ, Gerard RD et al. FoxO transcription factors activate Akt and attenuate insulin signaling in heart by inhibiting protein phosphatases. Proc Natl Acad Sci USA 2007; 104: 20517-20522.

38. Mu J, Brozinick JT Jr, Valladares O, Bucan M, Birnbaum MJ. A role for AMP-activated protein kinase in contraction- and hypoxia-regulated glucose transport in skeletal muscle. Mol Cell 2001; 7: 1085-1094.
39. Feng X, Li J, Liu J, Jin M, Liu X, Du H et al. Protective effect of FK506 on myocardial ischemia/reperfusion injury by suppression of $\mathrm{CaN}$ and ASK1 signaling circuitry. Cardiovasc Toxicol 2011; 11: 18-27.

40. Felkin LE, Narita T, Germack R, Shintani Y, Takahashi K, Sarathchandra P et al. Calcineurin splicing variant calcineurin Abeta1 improves cardiac function after myocardial infarction without inducing hypertrophy. Circulation 2011; 123: 2838-2847.

41. Bousette N, Chugh S, Fong V, Isserlin R, Kim KH, Volchuk A et al. Constitutively active calcineurin induces cardiac endoplasmic reticulum stress and protects against apoptosis that is mediated by alpha-crystallin-B. Proc Natl Acad Sci USA 2010; 107 : $18481-18486$.

42. Han J, Pan XY, Xu Y, Xiao Y, An Y, Tie L et al. Curcumin induces autophagy to protect vascular endothelial cell survival from oxidative stress damage. Autophagy 2012; 8 . 812-825.

43. Harhaji-Trajkovic L, Vilimanovich U, Kravic-Stevovic T, Bumbasirevic V, Trajkovic V. AMPK-mediated autophagy inhibits apoptosis in cisplatin-treated tumour cells. $\mathrm{J}$ Cell $\mathrm{Mol}$ Med 2009; 13: 3644-3654.

44. Nakamura T, Lipton SA. Cell death: protein misfolding and neurodegenerative diseases. Apoptosis 2009; 14: 455-468.

45. Fischer F, Hamann A, Osiewacz HD. Mitochondrial quality control: an integrated network of pathways. Trends Biochem Sci 2012; 37: 284-292.

46. Inoki K, Zhu T, Guan KL. TSC2 mediates cellular energy response to control cell growth and survival. Cell 2003; 115: 577-590.

47. Gwinn DM, Shackelford DB, Egan DF, Mihaylova MM, Mery A, Vasquez DS et al. AMPK phosphorylation of raptor mediates a metabolic checkpoint. Mol Cell 2008; 30: 214-226.

48. Ermak G, Davies KJ. Calcium and oxidative stress: from cell signaling to cell death. $\mathrm{Mol}$ Immunol 2002; 38: 713-721.

49. Ghislat G, Patron M, Rizzuto R, Knecht E. Withdrawal of essential amino acids increases autophagy by a pathway involving $\mathrm{Ca} 2+/$ calmodulin-dependent kinase kinase-beta (CaMKK-beta). J Biol Chem 2012; 287: 38625-38636.

50. Dwivedi M, Song HO, Ahnn J. Autophagy genes mediate the effect of calcineurin on life span in C. elegans. Autophagy 2009; 5: 604-607.

51. Inoki K, Li Y, Zhu T, Wu J, Guan KL. TSC2 is phosphorylated and inhibited by Akt and suppresses mTOR signalling. Nat Cell Biol 2002; 4: 648-657.

52. Wu YT, Tan HL, Huang Q, Ong CN, Shen HM. Activation of the PI3K-Akt-mTOR signaling pathway promotes necrotic cell death via suppression of autophagy. Autophagy 2009; 5 : 824-834.

53. Tao L, Xie Q, Ding YH, Li ST, Peng S, Zhang YP et al. CAMKII and Calcineurin regulate the lifespan of Caenorhabditis elegans through the FOXO transcription factor DAF-16. Elife 2013; 2: e00518.

54. Lara-Pezzi E, Winn N, Paul A, McCullagh K, Slominsky E, Santini MP et al. A naturally occurring calcineurin variant inhibits FoxO activity and enhances skeletal muscle regeneration. J Cell Biol 2007; 179: 1205-1218.

55. Sengupta A, Molkentin JD, Yutzey KE. FoxO transcription factors promote autophagy in cardiomyocytes. J Biol Chem 2009; 284: 28319-28331.

56. Lu J, Wu DM, Zheng YL, Hu B, Zhang ZF, Shan Q et al. Quercetin activates AMP-activated protein kinase by reducing $\mathrm{PP} 2 \mathrm{C}$ expression protecting old mouse brain against high cholesterol-induced neurotoxicity. J Pathol 2010; 222: 199-212.

57. Wu Y, Song $\mathrm{P}, \mathrm{Xu}$ J, Zhang M, Zou MH. Activation of protein phosphatase $2 \mathrm{~A}$ by palmitate inhibits AMP-activated protein kinase. J Biol Chem 2007; 282: 9777-9788.

58. Liu Q, Busby JC, Molkentin JD. Interaction between TAK1-TAB1-TAB2 and RCAN1calcineurin defines a signalling nodal control point. Nat Cell Biol 2009; 11: 154-161.

59. Tan X, Li J, Wang X, Chen N, Cai B, Wang G et al. Tanshinone IIA protects against cardiac hypertrophy via inhibiting calcineurin/NFATc3 pathway. Int J Biol Sci 2011; 7: 383-389.

(c) (i) (2) Cell Death and Disease is an open-access journal published by Nature Publishing Group. This work is licensed under a Creative Commons Attribution-NonCommercialShareAlike 3.0 Unported License. To view a copy of this license, visit http://creativecommons.org/licenses/by-nc-sa/3.0/ 